\title{
Survey of Bone Fracture Detection Techniques
}

\author{
Nathanael .E. Jacob \\ Department of $E$ \& Tc. \\ Vishwakarma Institute of \\ Technology \\ City- Pune, Maharashtra. \\ Country- India
}

\author{
M.V. Wyawahare \\ Department of E \& Tc. \\ Vishwakarma Institute of \\ Technology \\ City- Pune, Maharashtra. \\ Country- India
}

\begin{abstract}
This paper deals with techniques that have been used for bone fracture detection in the past few years. The authors have made attempts to survey papers from different modalities. This led us to study techniques that have been applied to images obtained from different modalities like X-ray, CT, MRI and ultrasound. The methods have been documented in a way that aids ease of interpretation. The paper is the first of its kind to survey fracture detection techniques across different modalities. The study will help the reader in designing computer aided diagnosis (CAD) systems in the field of medical imaging.
\end{abstract}

\section{General Terms}

Medical imaging, machine vision, biomedical image analysis.

\section{Keywords}

Survey, bone fracture, CAD, bone segmentation.

\section{INTRODUCTION}

The field of medical imaging has been witnessing advances not only in acquisition of medical images but also in its techniques of interpretation. In particular the research is on to interpret and diagnose ailments from medical images with minimum aid from medical experts [1]. Such systems called Computer aided diagnosis (CAD) systems can prove very useful to analyze large volumes of medical data, as well as improve the accuracy of interpretation while reducing time for diagnosis [2][3]

The following study explains the methods involved in designing $\mathrm{CAD}$ systems for bone fracture detection. The section has been divided into 4 sub-sections based on X-ray, CT, MRI and Ultrasound modalities. A total of 12 general techniques (which includes 7 techniques for X-ray images, 3 for CT, 1 for MRI and 1 for ultrasound images) from 29 papers have been surveyed.

\section{METHODS}

The section has been organized based on the modality to which the method has been applied. This will help the reader in understanding the potential and amount of research that have been carried in that field. An attempt has been made in providing vivid and crisp technical details of each paper, for the benefit of researchers new to this field.

\subsection{X-RAY}

\subsubsection{Calculating bone alignment}

[4] Y Jia and Y Jiang present a method that outlines fractured bones in an X-ray image of a patient's arm within casting materials, and displays the alignment between the fractured bones. A geodesic active contour model with global constraints is applied to segment the bone region. A prior shape is collected and used as a global constraint of our model. A maximum-likelihood function is derived to provide feedback for each evolving process. Experimental results show that the method produces the outlines of the fractured bones on the low contrast X-ray images robustly and accurately.

\subsubsection{Mathematical morphology}

[5] JIAN LIANG et al. have proposed morphological method to identify fractures in tibia bones. Before segmentation, the original image is dynamically divided into several intervals to help find out the smallest interval with the target. The small regions are then automatically thresholded using Otsu method .To promote the accuracy of segmentation and to avoid over or under segmentation, the segmentation result obtained is examined using statistical method. Depending on the test results the segmented image is adjusted .After the second segmentation, the steps of verification and adjustment are required to repeat till the test result conforms to any one of the stopping conditions. When the segmentation is finished, the target image will no long have tough areas.

This is followed by mathematical morphology to extract the target border and cover the boundary of fractures. Then by superposing the target border image and covering the extracted skeleton, the precise location of fractures can be recognized.

\subsubsection{Gradient analysis}

[6] Martin Donnelley et al. developed a method of automatically detecting fractures in long bones. First the edges are extracted from the x-ray image using a non-linear anisotropic diffusion method (the affine morphological scale space) that smoothes the image without losing critical information about the boundary locations within the image. Then a modified Hough transform with automatic peak detection is used to determine parameters for the straight lines that best approximate the edges of the long bones. The parameters used to approximate the long bone edges are then used for centreline approximation, diaphysis segmentation and fracture detection in the segmented region. 


\subsubsection{Classifiers}

7.

Neck Shaft angle [17] [11] [13]

[7] Syiam et al. have proposed an adaptive interface agent, called the AdAgen that collaborates with trained agents using neural network to build the software interface agent to detect fractures in long bone. The simulation results have shown how the neural network of the collaborating agents can help maintain the performance for automatic detection of fractures in leg radiograph.

[8]- [19] describe classifiers implemented for fracture detection. They are listed below:-

\subsubsection{Standard Classifiers}

1. $\quad$ BPNN [8] [9]

2. ANN [10] [7]

3. Bayesian, Bayes [11] [12] [13]

4. Gini-SVM [12]

5. Probabilistic SVM [12]

6. SVM [8] [9] [13] [14] [15] [16]

7. Hierarchy of SVM (SVM,SVM+ ,H-SVM,H-SVM) [14]

8. NB [8] [9] [14]

9. Binary/Linear Classifier [17] [18] [13] [19]

where, BPNN = Feed Forward Back Propagation Neural Networks, ANN = Artificial Neural network, SVM = Support Vector Machine Classifiers and NB = Naïve Bayes Classifiers

\subsubsection{Hybrid Classifiers}

$\begin{array}{ll}\text { 1. } & \text { BPNN and SVM [8] } \\ \text { 2. } & \text { BPNN and NB [8] } \\ \text { 3. } & \text { SVM and NB [8] } \\ \text { 4. } & \text { BPNN, SVM and NB [8] [9] } \\ \text { 5. } & \text { Bayesian and SVM [13] } \\ \text { 6. } & \text { NSA and Bayesian [13] } \\ \text { 7. } & \text { NSA and SVM [13] }\end{array}$

where NSA (Neck shaft angle) represents a binary classifier adopted from the paper [17].

The features used by one or more of the above classifiers are as follows:-
1. Shape parameters [19]
2. Appearance parameters [19]
3. Texture Features ( Contrast, Homogeneity, Energy, Entropy, Mean, Variance, Standard Deviation, Correlation ) [8] [9] [18] [13] [16] [19]
4. Gabor orientation (GO) [8] [9] [12] [14]
5. Markov Random Field (MRF) [8] [9] [11] [12]
6. Intensity Gradient Direction (IGD) [15] [8] [9] [11]
[12] [14]

[19] Martin G Roberts et al. investigated the use of classification techniques to detect and quantify vertebral fractures due to osteoporosis in dual X-ray absorptiometry (DXA) images of the spine.

\subsubsection{Using vertical integral projection}

[20] ZHENG wei et al. deal with the problem of automatic interpretation of fracture injury site in femur bones by converting it to bone shape identification problem. Depending on the shape of the bone segment, the fractures locations can be identified as - the proximal, middle or the distal part of femur bone. The algorithm conducts vertically integral projection for each pre-processed bone region in X-ray image, and combines the projection curves. After that, the Muller AO coding standing for the fracture injure site is judged based on analysis of subsection variances of curves. If the variance ratio is greater than a fixed value, the program output a numeric code " 1 ", if the variance ratio is less than a fixed value, the program output a numeric code " 3 ". Otherwise, the program output a numeric code " 2 ". Then the fracture injury site is automatically interpreted according to the code matching rules

\subsubsection{Using discrete wavelet transform}

[21] Rebecca Smith et al. present a fracture detection method for the pelvic ring based on Discrete Wavelet Transform. DWT is applied to windows extracted from the extracted from the ring as defined by prior automated region segmentation. The chosen wavelet coefficient is used to reconstruct an image that highlights the bone boundary. This is followed by morphological operations on its binary image. The bone boundaries of the ring are then traced using the 8neighborhood of each edge pixel and returned as a matrix of pixel positions. If there is no fracture, the window will contain a single uninterrupted boundary; otherwise there will be multiple boundaries depending on the types and number of fractures.

\subsubsection{By minimization of fuzzy index measure}

[22] The paper introduces a procedure for crack detection in $\mathrm{X}$-ray image, which is based on the minimization of a fuzzy measure. The image histogram is divided into three fuzzy subsets using iterative approach to obtain subsets parameters. The obtained parameters were used as initial estimates and each pixel in the fuzzy regions were classified as belonging to one of the sub-sets by minimizing the fuzzy index. After segmenting the image into three regions, the background and skin regions are removed to detect the cracks in the bone region.

A binary image thus obtained contains cavities or holes. A hole-filling step utilizing the morphological operation is then applied to the binary image to fill these spots and create a temporary image. The temporary image is subtracted by the original binary image to isolate the small spots. Morphological filtering functions (erosion followed by dilation) are then used to screen noise or undesirable spots using the iteration number as an operational parameter. The morphological operation can eliminate or maintain the spots on the image according to their area size. This is done to identify possible infestation sites among small spots segmented from the binary image. The output of this process is a binary image containing the crack 


\section{$2.2 \mathrm{CT}$}

\subsubsection{Using boundary tracing}

[23] Fracture detection is performed on the basis of the results of a prior pelvic bone segmentation algorithm - registered active shape model (RASM).The extracted bone boundaries are utilized to create a series of adaptive windows. Later a 2D stationary wavelet transform (SWT) is applied to each adaptive window to test the contour discontinuities in each window using boundary tracing. If there is a contour discontinuity in a window, then it is considered as a potential bone fracture.

[24] Jie $\mathrm{Wu}$ et al. have further developed their method by measuring the displacement between the fractured bones. It helps in detecting both major and minor fractures in pelvic bones

\subsubsection{Using two stage identification process}

[25] This paper proposes an automated method of pelvic fracture detection from volumetric CT images. A coarse-tofine strategy is adopted for that purpose. During the coarse fracture localization phase, a potential region containing the fracture is identified first using average intensity and sum of mean curvatures for valleys. In the precise detection phase, the above region is modelled as a weighted graph and a fracture is modelled as a minimum cut in this graph. An additional localizing algorithm models the same fracture as a valley, based on the signs of the mean and Gaussian curvature. A joint decision process based on the output of the above algorithms is used to identify the fracture voxels.

[26] In the first phase, the hairline fractures are coarsely localized using statistical correlation and by exploiting the bilateral symmetry of the human mandible. In the second phase, the fractures are precisely identified and highlighted using a Markov Random Field (MRF) modelling approach coupled with Maximum A Posterior probability (MAP) estimation. Gibbs sampling is used to maximize the posterior probability.

\subsubsection{Weighted flow network}

Since a hairline fracture is essentially a discontinuity in the bone contour this paper [27] models it as a minimum cut in an appropriately weighted flow network. The flow network is constructed based on the geometry of the human mandible and some prior knowledge of the fracture pattern. A simple capacity function is used to compute the edge weights. The Edmonds-Karp refinement of the classical Ford-Fulkerson algorithm is employed on the 2D flow network to obtain a minimum cut. The minimum cut correctly identifies a fracture in the proposed 2D flow network.

\subsection{MRI}

\subsubsection{Using finite element analysis}

[28] Victor Ortiz et al. developed an algorithm that allows detection of skull fractures by finite element method. The paper presents a new, physics-based deformable model for tracking fractures using image matching. The model results from the minimization of a deformation field simultaneously satisfying the constraints of an elastic body and a local image similarity measure. The model provides us with a physically realistic deformation field and also allows us to inspect the characteristics of the deformed objects. This can be very useful for the inspection of stresses induced by the fracture of skull on their surroundings. With the development of this paper the efficiency of the Method of Finite Element in the segmentation of images has been shown.

\subsection{Ultrasound}

\subsubsection{Local phase based technique}

In this paper [29], Ilker Hacihaliloglu et al. present an intensity invariant three dimensional (3D) local image phase features, obtained using 3D Log-Gabor filter banks, for extracting ridge like features (similar to those that occur at soft tissue and bone interfaces) from 3D US (ultrasound) volumes. Specifically, they constructed a 3D local phase symmetry measure which produces strong responses at bone surfaces and suppresses responses elsewhere. They quantitatively investigated the accuracy of their technique in localizing bone surfaces and assessed the technique's ability to resolve displaced bone fragments. The study is therefore the first of its kind to show that bone surfaces and fractures can be accurately localized using local phase features computed directly from 3D ultrasound image volumes.

\section{CONCLUSIONS}

The fracture detection techniques discussed above have been applied to different bones in the human body. This is illustrated in Fig 1 below.

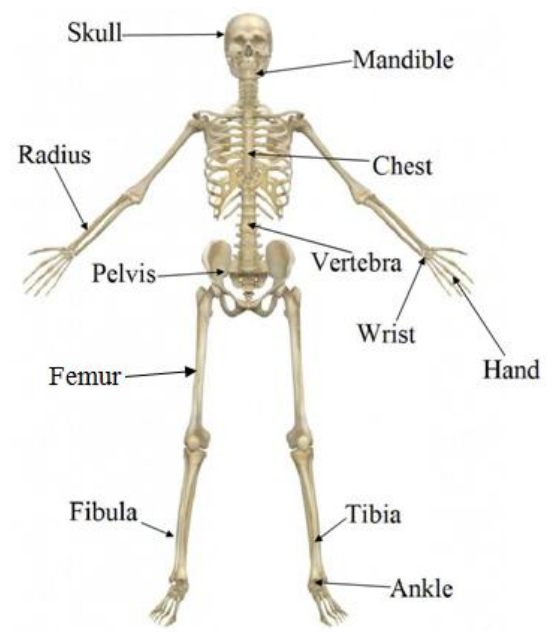

Fig 1: Bones on which fracture detection techniques have been surveyed.

Among the four modalities discussed (X-ray, CT, MRI, Ultrasound), X-ray diagnosis is commonly used for fracture detection unless the fracture is complicated (E.g. stress fractures) in which case a CT,MRI or ultrasound may be needed for further diagnosis and operation [30].

Among the fracture detection techniques discussed, fracture detection using classifiers in X-ray images appears promising. However there is a need to accurately detect fractures using minimum and computationally less expensive features and classifiers. Features that complement each other should be used. This can be achieved by performing relevance and redundancy analysis of classifiers [31], where optimal features can be selected from the original high dimensional feature set. This will help in achieving higher accuracy and better computational complexity.

Based on the study conducted, a major challenge in fracture detection systems is designing CAD systems that can handle fractures in any bone of the body for a given modality. 


\section{ACKNOWLEDGMENTS}

We would like to thank Mr.Ragvendra M.A.N.S from NIT, Suratkal, India for providing us with the materials that needed to be studied for the successful completion of this paper.

\section{REFERENCES}

[1] Giger, Maryellen L., Nico Karssemeijer, and Samuel G. Armato III. "Computer-aided diagnosis in medical imaging." IEEE Trans. Med. Imaging 20.12 (2001): 1205-1208.

[2] Umadevi, N., and S. N. Geethalakshmi. "Enhanced Segmentation Method for bone structure and diaphysis extraction from x-ray images." International Journal of Computer Applications 37.3 (2012): 30-36.

[3] Berbaum, Kevin S., et al. "Gaze dwell times on acute trauma injuries missed because of satisfaction of search." Academic radiology 8.4 (2001): 304-314.

[4] Jia,Y.,and Y.Jiang. "Active contour model with shape constraints for bone fracture detection." Computer Graphics, Imaging and Visualisation, 2006 International Conference on. IEEE, 2006.

[5] Liang, Jian, et al. "Fracture identification of X-ray image." Wavelet Analysis and Pattern Recognition (ICWAPR), 2010 International Conference on. IEEE, 2010.

[6] Donnelley, Martin William. Computer aided long-bone segmentation and fracture detection. Diss. Flinders University, Faculty of Science and Engineering., 2008.

[7] Syiam, Mostafa, Mostafa Abd El-Aziem, and Mohamed El-Menshawy. "AdAgen: Adaptive Interface Agent for X-Ray Fracture Detection." International Journal of Computing \& Information Sciences 2.3 (2004).

[8] Mahendran, S. K., and S. Santhosh Baboo. "Ensemble Systems for Automatic Fracture Detection." IACSIT International Journal of Engineering and Technology, Vol. 4, No. 1, February 2012.

[9] Mahendran, S. K., and S. Santhosh Baboo. "An Enhanced Tibia Fracture Detection Tool Using Image Processing and Classification Fusion Techniques in XRay Images." (2011).

[10] Eksi, Z., E. Dandil, and M. Cakiroglu. "Computer aided bone fracture detection." Signal Processing and Communications Applications Conference (SIU), 2012 20th. IEEE, 2012.

[11] Lim, Sher Ee, et al. "Detection of femur and radius fractures in x-ray images."Proc. 2nd Int. Conf. on Advances in Medical Signal and Info. Proc. 2004.

[12] Lum, Vineta Lai Fun, et al. "Combining classifiers for bone fracture detection in X-ray images." Image Processing, 2005. ICIP 2005. IEEE International Conference on. Vol. 1. IEEE, 2005.

[13] Yap, Dennis Wen-Hsiang, et al. "Detecting femur fractures by texture analysis of trabeculae." Pattern Recognition, 2004. ICPR 2004. Proceedings of the 17th International Conference on. Vol. 3. IEEE, 2004.

[14] He, Joshua, Wee Leow, and Tet Howe. "Hierarchical classifiers for detection of fractures in $\mathrm{x}$-ray images." Computer Analysis of Images and Patterns. Springer Berlin/Heidelberg, 2007.

[15] Peng, Tian Tai. "Detection of Femur Fractures in X-ray images." Master of Science Thesis, National University of Singapore (2002).
[16] Lee, Sooyeul, et al. "The Preliminary Study of Differentiating Osteoporotic Fractured Group from Nonfractured Group." Engineering in Medicine and Biology Society, 2005. IEEE-EMBS 2005. 27th Annual International Conference of the. IEEE, 2006.

[17] Tian, Tai, et al. "Computing neck-shaft angle of femur for x-ray fracture detection." Computer Analysis of Images and Patterns. Springer Berlin/Heidelberg, 2003.

[18] Chai, Hum Yan, et al. "Gray-level co-occurrence matrix bone fracture detection." American Journal of Applied Sciences 8.1 (2011): 26-32.

[19] Roberts, Martin G. Automatic detection and classification of vertebral fracture using statistical models of appearance. Diss. the University of Manchester, 2008.

[20] Wei, Zheng, and Zhang Liming. "Study on recognition of the fracture injure site based on X-ray images." Image and Signal Processing (CISP), 2010 3rd International Congress on. Vol. 4. IEEE, 2010.

[21] Smith, Rebecca, et al. "Detection of fracture and quantitative assessment of displacement measures in pelvic X-RAY images." Acoustics Speech and Signal Processing (ICASSP), 2010 IEEE International Conference on. IEEE, 2010

[22] Linda, C. Harriet, and G. Wiselin Jiji. "Crack detection in X-ray images using fuzzy index measure." Applied Soft Computing 11.4 (2011): 3571-3579.

[23] $\mathrm{Wu}, \mathrm{Jie}$, et al. "Fracture detection in traumatic pelvic CT images." International Journal of Biomedical Imaging Volume 2012, Article ID 327198.

[24] $\mathrm{Wu}$, Jie, et al. "Fracture detection and quantitative measure of displacement in pelvic CT images." Bioinformatics and Biomedicine Workshops (BIBMW), 2011 IEEE International Conference on. IEEE, 2011

[25] Chowdhury, Ananda S., et al. "Automated detection of pelvic fractures from volumetric CT images." Biomedical Imaging (ISBI), 2012 9th IEEE International Symposium on. IEEE, 2012.

[26] Chowdhury, A. S., et al. "Hairline fracture detection using MRF and Gibbs sampling." Applications of Computer Vision, 2007. WACV'07. IEEE Workshop on. IEEE, 2007.

[27] Chowdhury, Ananda S., et al. "Detection of hairline mandibular fracture using max-flow min-cut and Kolmogorov-Smirnov distance." Biomedical Imaging: From Nano to Macro, 2011 IEEE International Symposium on. IEEE, 2011.

[28] Ortiz, Victor, Cornelio Yanez, and Isaac Chairez. "Skull Fractures Detection by Finite Element Method." Computer Science and Engineering (2007): 100.

[29] Hacihaliloglu, Ilker, et al. "Bone segmentation and fracture detection in ultrasound using 3D local phase features." Medical Image Computing and ComputerAssisted Intervention-MICCAI 2008 (2008): 287-295.

[30] http://www.bupa.co.uk/individuals/healthinformation/directory/f/fractures\#textBlock206553

[31] Duangsoithong, Rakkrit, and Terry Windeatt. "Relevance and redundancy analysis for ensemble classifiers." Machine Learning and Data Mining in Pattern Recognition. Springer Berlin Heidelberg, 2009. 206-220. 